INVESTIGACIÓN

Recibido: 02/01/2019 --- Aceptado: 22/02/2019 --- Publicado: 15/06/2019

\title{
LA PLANIFICACIÓN ESTRATÉGICA DE LA COMUNICACIÓN EN LA ERA DIGITAL. LOS CASOS DE ESTUDIO DE WALLAPOP, WESTWING Y FOTOCASA
}

\section{Communication strategies in the new economy: the case studies of Wallapop, Westwing and Fotocasa}

\author{
(iD) Patricia Coll Rubio': Universitat Ramon Llull. Barcelona. España. \\ pcollr@uoc.edu
}

iD Josep Lluís Micó2: Universitat Ramon Llull. Barcelona. España. josepLluisMS@blanquerna.url.edu

\section{RESUMEN}

Las estrategias comunicativas, especialmente en la nueva economía, evolucionan de forma cada vez más acelerada, para adaptarse al complejo y cambiante escenario de la interconectada sociedad red.

Partiendo de la hipótesis de la existencia de un planteamiento de la estrategia de comunicación $360^{\circ}$, heredero del enfoque propio de la comunicación integrada de marketing, el artículo describe las estrategias de comunicación de tres marcas nativas digitales de referencia en Barcelona, sede del Mobile World Congress, donde se ha desarrollado un contexto interconectado de startups y marcas nativas digitales.

Los resultados de esta investigación provienen de tres casos de estudio (Wallapop, Westwing y Fotocasa), que muestran que su planificación estratégica consta de cuatro fases (investigación, planificación, ejecución y evaluación), coincidentes con el modelo RACE de Marston. La estrategia comunicativa que constatan estos casos de estudio pone el foco en el corto plazo, con una perspectiva growth hacker, característica del ecosistema startup, que aprovecha las sinergias entre acciones de publicidad y relaciones públicas. Para llevarlas a cabo estas acciones de comunicación, las marcas nativas digitales buscan sinergias entre acciones, utilizando todas las herramientas de investigación y medición a su alcance, que les permiten experimentar de forma controlada y tomar decisiones basadas en datos, en un proceso de aprendizaje continuo.

\footnotetext{
1 Patricia Coll Rubio es Doctora en Comunicación. Profesora colaboradora de la Universitat Ramon Llull, la Universitat Oberta de Catalunya y la Escuela Superior de Relaciones Públicas-UB.

${ }^{2}$ Josep Lluís Micó es Catedrático de Periodismo. Vicedecano de la Facultat de Comunicació i Relacions Internacionals Blanquerna (Universitat Ramon Llull).
} 
Coll Rubio, P., y Micó, J. L. La planificación estratégica de la comunicación en la era digital. Los casos de estudio de Wallapop, Wetswing y Fotocasa

PALABRAS CLAVE: comunicación integrada de marketing - nueva economía publicidad - relaciones públicas - marketing digital- growth hacking- estrategia de comunicación- comunicación 360.

\begin{abstract}
Communication strategies, especially in the new economy, evolve in an increasingly accelerated way, to adapt to the complex and changing scenario of the interconnected network society.

Based on the hypothesis of the existence of an approach to communication strategy $360^{\circ}$, heir to the integrated marketing communication approach, the article describes the communication strategies of three digital brands in Barcelona. The city is headquarters of the Mobile World Congress, with an interconnected ecosistem of startups and digital native brands.

The results of this research come from three case studies (Wallapop, Westwing and Fotocasa), which show that its strategic planning consists of four phases (research, planning, execution and evaluation), coinciding with the Marston RACE model. The communicative strategy observed in these case studies focuses on the short term, with a growth hacker perspective, characteristic of the startup ecosystem, which takes advantage of the synergies between publicity and public relations actions. To carry out these communication actions, the digital native brands seek all the research and measurement tools at their disposal, which allow them to experiment in a controlled way and make decisions based on data, in a process of continuous learning and taking advantage of synergies between coordinated actions.
\end{abstract}

KEY WORDS: integrated marketing communication - new economy - advertising public relations - digital marketing - growth hacking - communication strategy - 360 communication.

\title{
PLANIFICAÇÃO ESTRATÉGICA DA COMUNICAÇÃO NA ERA DIGITAL. OS CASOS DOS ESTUDOS DE WALLAPOP, WESTWING E FOTOCASA
}

\section{RESUME}

As estratégias comunicativas, especialmente na nova economia, evolucionam de forma cada vez mais acelerada, para adaptar-se ao complexo e que tanto muda no cenário da interconectada redes sociais. Partindo da hipótese da existência de um planejamento da estratégia de comunicação 360 graus, herdeiro do enfoque próprio da comunicação integrada de marketing, o artigo descreve as estratégias de comunicação de três marcas nativas digitais de referência em Barcelona, sede do Mobile WORLD Congress, onde foi desenvolvido um contexto interconectado de startups e marcas nativas digitais. Os resultados desta investigação provem de três casos de estudo Wallapop, Westwing e Fotocasa, que mostram que sua planificação estratégica consta de quatro fases:

Vivat Academia. Revista de Comunicación. 15 junio 2019 /15 septiembre 2019, n 147, 125-138 
Coll Rubio, P., y Micó, J. L. La planificación estratégica de la comunicación en la era digital. Los casos de estudio de Wallapop, Wetswing y Fotocasa

(investigação, planificação, execução e avaliação), coincidentes com o modelo RACE de Marston. A estratégia comunicativa que constatam estes casos de estudo põe o foco no curto prazo, com uma perspectiva growth hacker, característica do ecossistema startup, que aproveita as sinergias entre ações de publicidade e relações públicas. Para leva-las à cabo estas ações de comunicação, as marcas nativas digitais buscam sinergias entre ações, utilizando todas as ferramentas de investigação e medição a seu alcance, que os permitem experimentar de forma controlada e tomar decoes baseadas em dados, em um processo de aprendizagem continuo.

PALAVRAS CHAVE: comunicação integrada de marketing - nova economia publicidade - relações públicas - marketing digital - growth hacking - estratégia de comunicação - comunicação 360.

\section{Como citar el artículo:}

Coll Rubio, P., y Micó, J. L. (2019). La planificación estratégica de la comunicación en la era digital. Los casos de estudio de Wallapop, Wetswing y Fotocasa. [Communication strategies in the new economy: the case studies of Wallapop, Westwing and Fotocasa]. Vivat Academia. Revista de Comunicación, 147, 125-138. http://doi.org/10.15178/va.2019.147.125-138

Recuperado de http://www.vivatacademia.net/index.php/vivat/article/view/1147

\section{INTRODUCCIÓN}

Las reflexiones de este estudio se enmarcan en un momento en el que las estrategias de comunicación de todo tipo de organizaciones se están redefiniendo, en línea con los cambios en los usos de la audiencia y los cambios en el ecosistema económico global. Consideramos que el estudio de las estrategias de comunicación por parte de empresas nativas digitales (Prensky, 2001) tiene valor en la investigación en el campo de la comunicación, en cualquiera de sus disciplinas.

\subsection{La planificación estratégica de la comunicación}

El concepto de estrategia de comunicación se define como "una orientación de intervención o un enfoque de acción para una problemática que hay que resolver o para un proyecto particular por realizar" (Xifra, 200, p. 10). El modelo de planificación estratégica de la comunicación más ampliamente aceptada (Matilla, p. 406) es el proceso de toma de decisiones estratégicas definido por Marston (1963) bajo el acrónimo RACE, formado por cuatro etapas que se suceden en espiral: Research, Action, Communication.

La investigación es la primera fase del modelo RACE. De acuerdo con Cutlip y Center (2001) esta es la fase más difícil, ya que a menudo ni se sabe cómo plantearla y, además, cuenta con la oposición de quienes no la consideran necesaria. Además, su complejidad 
Coll Rubio, P., y Micó, J. L. La planificación estratégica de la comunicación en la era digital. Los casos de estudio de Wallapop, Wetswing y Fotocasa

ha ido en aumento con la irrupción de las tecnologías de la información, que permiten obtener datos masivos, denominados como big data (Braulio y Curto, 2015).

La segunda fase del modelo RACE (Marston, 1963) es la planificación, considerada como el proceso de las metas y objetivos y determinar la manera de lograrlos (Wilcox et al., 2012).

La tercera fase, de ejecución de las acciones de comunicación, se inscribe en la línea establecida por las dos fases precedentes, la investigación y la planificación.

Por último, la evaluación permite medir los más científicamente posible la consecución de los objetivos, para poder rectificar o incluso replantear la estrategia (Xifra, 2007). La Asociación de Empresas Consultoras en Comunicación y Relaciones Públicas (ADECEC) publicó en 2016 la Guía Práctica de la Medición, donde constata la importancia de esta fase y la necesidad de definir los indicadores clave para poder evaluar el éxito de cada acción de comunicación. (ADECEC, 2016, p. 7).

Matilla describe esta espiral "como una línea ascendente que, partiendo en su nacimiento de la investigación, avanza hacia la acción, se desplaza por la comunicación y, finalmente, desemboca y termina en la evaluación" (...) y la aportación de Marston (1963) en definir un modelo cerrado en el que "cada etapa conduce indefectiblemente a la siguiente, de modo que la última etapa (Evaluation), una vez superada, obligará a recurrir nuevamente a la primera (Research)" (Matilla, 2008, p. 74) De acuerdo con Wilcox, Cameron y Xifra, el modelo RACE, en definitiva, "es un proceso que puede considerarse como un ciclo sin fin" (Wilcox et al., 2012, p. 12).

\subsection{De la comunicación integrada al 'growth hacking'}

Como constatan las conclusiones del presente estudio, la estrategia comunicativa de las marcas nativas digitales (Prensky, 2001) sigue los principios de la Integrated Marketing Communications (IMC), ya que coordina acciones de disciplinas diversas, como el marketing, la publicidad y las relaciones públicas, para aprovechar sinergias (Schultz y Kitchen, 2000; Kliatchko, 2005; Schultz y Patti 2009).

El ámbito de la IMC analiza por qué los consumidores responden a algún tipo de comunicación de forma más positiva que otros y cómo las acciones de comunicación pueden coordinarse y aprovechar sinergias. En esa época, se plantea la necesidad de integrar de forma armoniosa todos los instrumentos del mix de comunicación en un conjunto unificado. Así, con independencia de la disciplina que se considere y de los instrumentos, medios o canales que se emplee, la marca hablará al mercado y a sus públicos con una sola voz. Con esta integración, se logra, además de coherencia, un mayor impacto. Antes de que surgiera el concepto de la IMC, autores de referencia en el 
Coll Rubio, P., y Micó, J. L. La planificación estratégica de la comunicación en la era digital. Los casos de estudio de Wallapop, Wetswing y Fotocasa

ámbito de la comunicación, como Marston, habían constatado ya esta realidad al señalar que "Las relaciones públicas y los programas publicitarios de una empresa están obviamente estrechamente conectados. ¿Por qué tienes una cierta imagen mental de una empresa en particular? ¿Porque conoces sus productos? ¿Porque has conocido a algunas de sus personas? Sí, pero también porque has visto publicidad". (Marston, 1963, p. 4). La IMC, de acuerdo con Kotler es, en definitiva, "un concepto según el cual una empresa integra y cuidadosamente coordina sus variados canales de comunicación para conseguir un mensaje claro, coherente y convincente sobre la empresa y sus productos" (Kotler, 2003, p. 131).

Los primeros teóricos que abordaron el impacto de Internet en el terreno del marketing se centraron principalmente en estudiar cómo sacar partido de un sitio web para la comunicación comercial y la venta a distancia. Pero en poco tiempo, Internet fue considerado mucho más que un canal de venta, llegando a ser considerado un nuevo medio de comunicación de masas y un soporte publicitario con gran proyección de futuro. De acuerdo con Meeker "La publicidad en la red no consiste tan sólo en anunciar y distribuir mensajes. También facilita las relaciones con los clientes, la creación de cibermarcas, proporciona servicios al consumidor, genera ventas electrónicas de marketing a la audiencia adecuada y logra crear una personalización de servicios para grandes masas de consumidores, así como un marketing directo e interactivo". (Meeker, 1997, pp. 47-48).

Con la irrupción de las redes sociales empieza a utilizarse el término web 2.0, que fue acuñado por O'Reilly (2005) para referirse a una segunda generación de la web que, gracias al uso intensivo de páginas dinámicas y aplicaciones conocidas como software social, se beneficia de la inteligencia colectiva para proporcionar servicios interactivos y donde los usuarios tienen control sobre la información.

Entre los factores que definen la web 2.0, Marquina destaca la importancia de las "aplicaciones en perpetua situación experimental o en pruebas (fase beta), o sea, en constante evolución". (Marquina, 2013, p. 12).

Otro factor esencial de la web 2.0, más allá de la tecnología, pero propiciado por ella, es "la nueva actitud" de los usuarios (Marquina, 2013, p. 12). Los públicos tienen un papel cada vez activo en los procesos comunicativos que se generan entre marca y clientes, ya que, de acuerdo con Martí (2010, p. 206), la web 2.0 "democratiza el conocimiento y la participación de los usuarios en el medio; incentiva la cocreación de los contenidos que se generan en ella; y permite el trasvase de estos contenidos entre los diferentes medios digitales interactivos al servicio del usuario".

Por todo ello, con la irrupción de Internet y las redes sociales, el concepto de IMC evoluciona hacia la comunicación $360^{\circ}$. Tal como destacan autores como Véliz (2006, p.

Vivat Academia. Revista de Comunicación. 15 junio 2019 /15 septiembre 2019, nº 147, 125-138 
Coll Rubio, P., y Micó, J. L. La planificación estratégica de la comunicación en la era digital. Los casos de estudio de Wallapop, Wetswing y Fotocasa

93) o Pintado y Sánchez (2009, p. 19), la comunicación $360^{\circ}$ debe caracterizarse por diálogo y la relación con el entorno de forma constante. Por tanto, tal como señala Sánchez Herrera (2012, p. 13) la comunicación $360^{\circ}$ es una evolución de la comunicación integrada de marketing, que combina "la imagen corporativa, la comunicación interna, las campañas publicitarias tradicionales, las relaciones públicas, la publicidad online, etc., siempre orientadas a conseguir el mismo objetivo".

Como evolución de este enfoque, en el seno del funcionamiento de estas marcas nativas digitales (Prensky, 2001) nace el denominado growth hacking (Ellis, 2010; Ellis y Brown, 2018), una pluridisciplina que se caracteriza por tener como objetivo el crecimiento: "un growth hacker es una persona cuyo verdadero norte es el crecimiento. (...) Debe tener la creatividad para descubrir maneras únicas de impulsar el crecimiento, además de probar o evolucionar las técnicas probadas por otras compañías" (Ellis, 2010).

\section{METODOLOGÍA}

La presente investigación cualitativa tiene por objeto describir el modelo de planificación estratégica de la comunicación de las marcas nativas digitales. Para ello, se escogen empresas cuyo departamento de marketing y comunicación esté ubicado en Barcelona y que marcas nativas digitales de referencia en su sector, partiendo de una entrevista en profundidad previa con el director ejecutivo de 4YFN Mobile World Capital, Esteban Redolfi.

Los casos de estudio seleccionados, que finalmente se presentan, al constatarse que ofrecían información suficiente para poder abordar la investigación son:

Wallapop: la primera app móvil española de compraventa geolocalizada, fundada en Barcelona en 2013. La plataforma centra su interés en los objetos de segunda mano, ya que permite a los usuarios poner a la venta aquello que ya no usan.

Westwing: fundada en Alemania en el año 2011, es la primera plataforma europea de comercio electrónico dedicada exclusivamente al sector Home\&Living. Westwing lidera el mercado español de venta de muebles.

Fotocasa: portal inmobiliario nacido en 2004 de la fusión de los portales Anuntis y Vivendum, que actualmente pertenece a Schibsted Spain, compañía de anuncios clasificados y ofertas de empleo más grande y diversificada de España.

La recogida de información en este estudio parte de la triangulación de los métodos de la observación, el análisis documental y la entrevista. La investigación consta de tres fases de entrevistas en profundidad sucesivas y complementarias, a los diferentes profesionales implicados en la estrategia de comunicación de las marcas de la nueva economía objeto de estudio y en su ejecución. Los informadores que participaron en

Vivat Academia. Revista de Comunicación. 15 junio 2019 /15 septiembre 2019, nº 147, 125-138 
Coll Rubio, P., y Micó, J. L. La planificación estratégica de la comunicación en la era digital. Los casos de estudio de Wallapop, Wetswing y Fotocasa

cada una de las tres rondas de entrevistas fueron escogidos por cada marca en función de las necesidades planteadas por la investigación.

Para cada caso de estudio se realizan tres fases de entrevistas. La primera es una entrevista en profundidad, vía cuestionario abierto, a las responsables de la estrategia de marketing y comunicación de cada una de las marcas objeto de estudio. La segunda fase de entrevistas, presenciales y en grupo, se realiza a las personas encargadas de la ejecución del plan de comunicación de cada una de ellas. Esta segunda fase, se realiza en el centro de trabajo del equipo de comunicación de cada una de las marcas e incluye una intensiva tarea de observación. La observación de campo permite "recorrer la escena" tanto antes como durante la realización de las entrevistas, para poder interpretar mejor la información que aportan los sujetos de estudio. Esta observación, en este caso durante tres años, se considera de largo recorrido teniendo en cuenta que se enmarca en el ecosistema de la nueva economía. En paralelo, la recogida de datos incluye, además de las anotaciones y registros de la observación y las entrevistas, la recopilación y análisis de más de 12.000 documentos.

Finalizada esta segunda fase, al considerarse que ya se cuenta con suficiente información relevante como para poder extraer conclusiones, se decide llevar a cabo una tercera ronda de entrevistas para poner en común toda la información recabada, como elemento de validación y actualización antes de dar por finalizado el trabajo de campo. Esta tercera fase, que se realiza vía telemática, consiste en un cuestionario que incluye los principales conceptos recogidos a partir de la realización de los tres casos de estudio.

\section{OBJETIVOS}

Este artículo pretende contribuir al conocimiento en el ámbito de las estrategias de comunicación, herederas de la comunicación integrada de marketing, a partir del análisis de tres casos prácticos de referencia en el ámbito de la nueva economía en Barcelona, sede de la feria internacional tecnológica Mobile World Congress y quinto hub europeo en volumen de startups, con más de 1.200 empresas tecnológicas emergentes instaladas en la ciudad y su entorno (Atómico, 2018).

La investigación permite también constatar que la metodología de los casos de estudio está especialmente indicada para el análisis de fenómenos recientes, y, por tanto, constituye una vía de análisis útil para el contexto cambiante en el que se desarrolla, cada vez más, el entorno comunicativo en la sociedad red (Castells, 2001).

\section{RESULTADOS}

Los resultados de la investigación se presentan en forma de tres casos de estudio, que se presentan siguiendo una misma estructura: la del modelo de planificación estratégica 
Coll Rubio, P., y Micó, J. L. La planificación estratégica de la comunicación en la era digital. Los casos de estudio de Wallapop, Wetswing y Fotocasa

de la comunicación RACE (Marston, 1963) que consta de cuatro fases: investigación, planificación, ejecución y evaluación.

\subsection{El caso de estudio de Wallapop}

Wallapop, la primera app móvil española de compraventa geolocalizada, se fundó en Barcelona en septiembre de 2013 con el objetivo de crear una alternativa de economía sostenible y adaptada a las nuevas formas de consumo, que se caracterizan por ser rápidas, locales y de fácil uso. La plataforma centra su interés principalmente en los objetos de segunda mano, ya que permite a los usuarios poner a la venta aquello que ya no usan para que otras personas que se encuentran a su alrededor puedan comprarlos. Actualmente, Wallapop cuenta con 20 millones de usuarios que realizan una media de 70.000 transacciones diarias de alguno de los más de 100 millones de productos subidos a la aplicación. La estrategia de comunicación de Wallapop sigue el modelo RACE (Marston, 1963), el esquema de planificación estratégica más ampliamente aceptado en el ámbito de la comunicación.

El estudio muestra, como se detalla a continuación, que la secuencia en la que se lleva a cabo la estrategia de comunicación de Wallapop consta de las cuatro etapas que abordaremos a continuación: investigación, planificación, ejecución y evaluación.

\subsubsection{La investigación en Wallapop}

La primera etapa de investigación, en el caso de Wallapop, es una etapa ineludible en toda planificación estratégica de la comunicación, ya que sus decisiones, previas al lanzamiento de cualquier acción futura, vienen siempre avaladas por datos, tal como constata triangulación de los datos obtenidos mediante la observación, el análisis documental y las sucesivas entrevistas en profundidad realizadas a su equipo de comunicación. En este sentido, como práctica habitual en esta fase de investigación, destaca la realización de test A/B, probando dos posibles propuestas comunicativas con el objetivo de comprobar cuál de ellas maximiza la conversión.

\subsubsection{La planificación en Wallapop}

La segunda etapa en la que se desarrolla la estrategia de comunicación de Wallapop es la planificación en la que se definen objetivos claros, cuantificables, ponderables, realistas y temporalizados para luego elegir los medios.

Para hacer frente al imperativo de la velocidad, los planes de comunicación en Wallapop se abordan a pocos meses vista. El equipo de comunicación de la startup considera como largo plazo la planificación semestral, medio plazo la trimestal y corto plazo la mensual. 
Coll Rubio, P., y Micó, J. L. La planificación estratégica de la comunicación en la era digital. Los casos de estudio de Wallapop, Wetswing y Fotocasa

\subsubsection{La ejecución en Wallapop}

La triangulación de los datos obtenidos mediante las técnicas de la observación participante, el análisis documental y las diferentes fases de entrevistas en profundidad realizadas muestra que la estrategia de comunicación de Wallapop se define como $360^{\circ}$, con una visión growth hacker que combina campañas de publicidad y acciones de publicity, comunicación de marketing e influencer marketing, combinando todos los canales posibles on y offline que, de forma combinada, permiten obtener mejores métricas en la fase de evaluación. En este sentido, la publicidad en televisión, financiada a través de acuerdos de media for equity, combinada con el marketing digital y la publicity, son la clave de la estrategia comunicativa de la startup.

\subsubsection{La evaluación en Wallapop}

La medición de resultados es un elemento clave en la estrategia de comunicación de Wallapop. Los datos cierran el círculo constantemente, llevan ineludiblemente a la acción $\mathrm{y}$, por tanto, son los que dirigen cualquier decisión que afecta a las acciones de comunicación de Wallapop y sirven además para aportar la información necesaria para la comunicación con otro de sus públicos clave, que son los inversores, para los que miden, sobre todo la conversión en descargas y usuarios activos. En este sentido, el plan de medios es dinámico y se va ajustando en función de los resultados obtenidos.

\subsection{El caso de estudio de Westwing}

Westwing.es pertenece al grupo Westwing Group $\mathrm{GmbH}$, fundado en Alemania en el año 2011 por la experta en decoración Delia Fischer, que supuso la creación de la primera plataforma europea de comercio electrónico dedicada exclusivamente al sector Home\&Living. Lidera el mercado español de venta de muebles. Cada 5 segundos Westwing vende un producto en todo el mundo y cerca del 50\% de los ingresos se genera a través de dispositivos móviles.

La estrategia de comunicación de Westwing sigue el modelo RACE (Marston, 1963), el esquema de planificación estratégica más ampliamente aceptado en el ámbito de la comunicación.

\subsubsection{La investigación en Westwing}

La triangulación de los datos obtenidos a través de las sucesivas entrevistas en profundidad realizadas durante la investigación al equipo de comunicación de la firma, el análisis documental de todo su archivo histórico y la observación realizada permite afirmar que la primera etapa de investigación es clave y viene determinada por la gran cantidad de datos que genera el e-commerce, que son analizados para maximizar las posibilidades de éxito de las acciones de comunicación. 
Coll Rubio, P., y Micó, J. L. La planificación estratégica de la comunicación en la era digital. Los casos de estudio de Wallapop, Wetswing y Fotocasa

\subsubsection{La planificación en Westwing}

La planificación en Westwing se realiza, de forma genérica, anualmente, pero las acciones se desarrollan de forma detallada por trimestres, para poder tener mayor capacidad de reacción. Sus objetivos, claros y medibles, están orientados claramente a aumentar la notoriedad de la marca entre su público objetivo, formado por compradores de decoración y sus prescriptores.

\subsubsection{La ejecución en Westwing}

La triangulación basada en el análisis documental, la observación y las entrevistas en profundidad constatan que Westwing apuesta claramente por la estrategia de generar contenido relevante en todo tipo de formatos para posicionarse como referente en su ámbito. Contamos con una redacción propia, un equipo editorial interno de periodistas y fotógrafos dedicados específicamente a contenidos, que difunden a diario a través del envío de una newsletter y en redes sociales.

La estrategia de comunicación de Westwing combina una potente estrategia de marketing de contenidos digital, con campañas de publicidad on y offline y acciones de relaciones públicas.

Las acciones se plantean y ejecutan de forma integrada, con visión $360^{\circ}$ y enfoque growth hacker, a través de la creatividad y de la sinergia entre canales para lograr el objetivo de máxima notoriedad.

\subsubsection{La evaluación en Westwing}

La cuarta fase de la estrategia de comunicación de Westwing es la evaluación que incluye el seguimiento de forma constante y a tiempo real de las acciones que se ejecutan, para evaluar el retorno de la inversión.

\subsection{El caso de estudio de Fotocasa}

El portal inmobiliario Fotocasa nació en 2004 de la fusión de dos portales, Anuntis y Vivendum. Actualmente pertenece a Schibsted Spain, compañía de anuncios clasificados y ofertas de empleo más grande y diversificada de España, que cuenta con otros portales como InfoJobs, Vibbo, Coches.net y Milanuncios, y que forma parte de la multinacional noruega Schibsted Media Group.

La estrategia de comunicación de Fotocasa sigue también el modelo RACE (Marston, 1963), con cuatro fases en su planificación estratégica.

\subsubsection{La investigación en Fotocasa}

Cualquier plan de comunicación en Fotocasa parte de la investigación, ya que tal como constata la triangulación, la mayoría de sus acciones de comunicación está basada en 
Coll Rubio, P., y Micó, J. L. La planificación estratégica de la comunicación en la era digital. Los casos de estudio de Wallapop, Wetswing y Fotocasa

datos, algunos de ellos obtenidos mediante pruebas consistentes en impactar sobre un porcentaje del público objetivo para poder medir previamente su efectividad.

\subsubsection{La planificación en Fotocasa}

En Fotocasa la planificación se construye a partir de la definición de objetivos de comunicación que se establecen anualmente, co alineados con los objetivos del negocio, medibles con indicadores clave entre los que destaca el share of voice.y con acciones que se detallan trimestralmente, Además, cuentan con un plan táctico ajustable a las necesidades del momento, con una toma de decisiones ágil y basada en datos.

\subsubsection{La ejecución en Fotocasa}

La ejecución del plan de comunicación de Fotocasa combina acciones de relaciones públicas, entre ellas gabinete de prensa, marketing de contenidos e influencer marketing, y publicidad, que se abordan de forma coordinada e integrada, con acciones tanto on como offline que se refuerzan mutuamente, con una visión $360^{\circ}$ y alineada con un enfoque growth hacker. Cualquier acción que se plantea se analiza teniendo en cuenta cómo se puede explotar desde cualquier área o cómo puede afectar al resto de áreas.

\subsubsection{La evaluación en Fotocasa}

El seguimiento del resultado de las acciones de comunicación se realiza de forma constante y se tiene en cuenta a la hora de planificar nuevas acciones, en consonancia con la espiral que describe el modelo RACE (Marston, 1963).

Así lo constata el análisis de los datos obtenidos mediante triangulación, en el que se detecta la utilización de métricas como el $P R$ Value y el top of mind mensual, así como el análisis del ROI y del SOV para poder valorar la eficacia de las acciones.

\section{CONCLUSIONES}

Los resultados del estudio muestran que la planificación estratégica de la comunicación en las marcas nativas digitales objeto de estudio (Wallapop, Westwing y Fotocasa) responde a un modelo de cuatro fases -investigación, planificación, ejecución y evaluación-, que coincide con el modelo RACE de Marston (1963).

Las particularidades que presenta la estrategia de comunicación de las marcas de la economía digital objeto de estudio derivan del funcionamiento del ecosistema económico en el que se encuentran inmersas, donde impera la velocidad y la toma de decisiones basada en datos. En este sentido, el estudio constata que la toma de decisiones es ágil y está basada en datos, provenientes tanto de la investigación previa como de la evaluación, con seguimiento a tiempo real de las acciones, creativas y

Vivat Academia. Revista de Comunicación. 15 junio 2019 /15 septiembre 2019, nº 147, 125-138 
Coll Rubio, P., y Micó, J. L. La planificación estratégica de la comunicación en la era digital. Los casos de estudio de Wallapop, Wetswing y Fotocasa

analíticas con un enfoque growth hacker heredero de la comunicación integrada de marketing, que se planifican y ejecutan con flexibilidad, siempre guiadas por objetivos cuantificables y medibles.

Las marcas nativas digitales objeto de estudio recurren tanto a la publicidad como a las relaciones públicas, tanto online como offline, con una presencia destacada de acciones de comunicación de marketing como el marketing de contenidos y el influencer marketing.

La estrategia de comunicación, por tanto, es integrada, con una visión $360^{\circ}$ que potencia sinergias entre las acciones que desarrolla en cuatro fases -investigación, planificación, ejecución y evaluación- para alcanzar sus objetivos, que son claros y medibles.

\section{REFERENCIAS}

ADECEC (2016). Guía Práctica de la Medición. Recuperado de http://www.adecec.com/pdf/adecec_presenta_la_guia_practica_de_la_medicion.pdf

Atómico (2018). The State of the European Tech Report. Recuperado de https://2018.stateofeuropeantech.com

Braulio, N y Curto, J. (2015). Customer analytics: mejorando la inteligencia del cliente mediante los datos. Barcelona: Editorial UOC.

Castells, M. (2001). La Galaxia Internet. Reflexiones sobre internet, empresa y sociedad. Barcelona: Plaza y Janés.

Ellis, S. (2010). Find a growth hacker for your startup. Startup Marketing. Recuperado de http://www.startup-marketing.com/where-are-all-the-growth-hackers/

Ellis, S., y Brown, M. (2018). El método Hacking Growth: Qué hacen compañias explosivas como Facebook, Airbnb y Walmart para ser líderes en el mercado. Barcelona: Penguin Random House.

Kliatchko, J. (2005). Towards a new definition of integrated marketing communications (IMC). International Journal of Advertising, 24(1), 7-34.

Kotler, P. (2003). Marketing Management. New Jersey: Prentice Hall.

Matilla, K. (2008). Los modelos de planificación estratégica en la teoría de las relaciones públicas. Barcelona: Editorial UOC.

Vivat Academia. Revista de Comunicación. 15 junio 2019 / 15 septiembre 2019, nº 147, 125-138 
Coll Rubio, P., y Micó, J. L. La planificación estratégica de la comunicación en la era digital. Los casos de estudio de Wallapop, Wetswing y Fotocasa

Marquina, J. (2013). Plan social media y community manager. Barcelona: Editorial UOC.

Marston, J. E. (1963). The nature of public relations. New York: McGraw-Hill.

Martí, J. (2010). Funny Marketing. Madrid: Wolters Kluwer.

Meeker, M. (1997). Internet Advertising Report. New York: Harper Collins.

O'Reilly, T (2005) What is Web 2.0. Design patterns and business model for the next generation of software. O'Reilly. Recuperado de http://www.oreilly.com/pub/a/web2/archive/what-is-web-20.html

Pintado, T., y Sánchez, J. (2009). Imagen corporativa. Influencia en la gestión empresarial. Madrid: ESIC Editorial.

Prensky, M. (2001) Digital Natives, Digital Immigrants. On the Horizon, 9(5), 1-6.

Sánchez Herrera, J. (2012). Nuevas tendencias en comunicación. Madrid: ESIC Editorial.

Schultz, D. E., y Kitchen, P. J. (2000). A response to 'Theoretical concept or management fashion'. Journal of Advertising Research, 40(5), 17-21.

Schultz, D. E., y Patti, C. H. (2009). The evolution of IMC: IMC in a customer-driven marketplace. Journal of Marketing Communications, 15(2-3).

Xifra, J. (2007). Tècniques de les relacions públiques. Barcelona: Editorial UOC.

Véliz, F. (2006). Cambio de mirada en las organizaciones: comunicación en 360. Revista Lationamericana de Comunicación, 93, 62-65.

Wilcox, D. L; Cameron, G. T; y Xifra, J. (2012). Relaciones Públicas: estrategias y tácticas. Madrid: Pearson Educación.

\section{AUTORES}

\section{Patricia Coll Rubio}

Doctora en Comunicación por la Facultat de Comunicació i Relacions Internacionals Blanquerna (URL). Es profesora colaboradora en la Universitat Oberta de Catalunya (UOC), la URL y la Escola Superior de Relacions Públiques-Universitat de Barcelona (ESRP-UB) y miembro del grupo de investigación STREAM de la URL; imparte clases de comunicación en universidades, escuelas de negocio y centros de formación. Colabora como periodista con El Pais Retina, The New Barcelona Post y Wolters Kluwer. 
Coll Rubio, P., y Micó, J. L. La planificación estratégica de la comunicación en la era digital. Los casos de estudio de Wallapop, Wetswing y Fotocasa

pcollr@uoc.edu

Orcid ID: https://orcid.org/0000-0001-7649-800X

Google Scholar: Google Scholar

\section{Josep Lluís Micó}

Catedrático de Periodismo de la Universitat Ramon Llull (URL) y vicedecano de la Facultat de Comunicació i Relacions Internacionals Blanquerna (URL). Ha liderado proyectos de investigación internacionales y es autor de decenas de artículos, capítulos y libros sobre comunicación y tecnología. Además, ha obtenido premios internacionales de periodismo, ensayo e investigación. Es analista de tecnología y tendencias en medios como La Vanguardia, Radio Nacional de España, NacióDigital, Diari de Girona, etc. josepLluisMS@blanquerna.url.edu

Orcid ID: https:// orcid.org/0000-0003-1191-226X

Google Scholar: Google Scholar 